\title{
Antibiotics Prescription Impact on Late Dental Implants Survival
}

\author{
Rawaa Younus Al-Rawee ${ }^{1 *}$, Sajid Khazaal Ameen ${ }^{2}$ \\ ${ }^{1}$ Department of Oral and Maxillofacial Surgery, Al-Salam Teaching Hospital, Mosul, Iraq \\ ${ }^{2}$ Clinical Pharmatist, Al-Shafa Hospital. Mosul, Iraq
}

\begin{abstract}
*Corresponding Author: Rawaa Younus Al-Rawee, Consultant Maxillo Facial Surger, Department of Oral and Maxillofacial Surgery, Al-Salam Teaching Hospital, Mosul, Iraq; Email: dr.rawarawi@yahoo.com
\end{abstract}

Received Date: 05-03-2021; Accepted Date: 26-03-2021; Published Date: 02-04-2021

Copyright $^{\odot} 2021$ by Al-Rawee RY, et al. All rights reserved. This is an open access article distributed under the terms of the Creative Commons Attribution License, which permits unrestricted use, distribution and reproduction in any medium, provided the original author and source are credited.

\begin{abstract}
Aims from this study is evaluating effect of antibiotics administration on dental implants failure in healthy patients underwent simple to moderate dental implant surgeries whether delayed or immediate (post-extraction) implantation. Complete assessment of the valuable or risky effects of oral antibiotics use with dental implant placement versus no antibiotic administration.

One hundred twenty (120) consecutive medically fit patients necessitate oral rehabilitation by dental implants in different tooth loss area. These patients were arbitrarily distributed to 3 diverse categories:

1. No Antibiotic Prescription (NOAB)

2. Preoperative and Postoperative Antibiotic use (PPAB) consisting of amoxicillin $1.5 \mathrm{~g} 1$ hour before surgery and $500 \mathrm{mg}$ three times per day; for 7 days following surgery

3. Postoperative Antibiotic Coverage (POAB) consisting of and $500 \mathrm{mg}$ three times per day; started after surgery and continued for 1 week after surgery.

Significant correlation are shown in this study analysis for three years follow-up periods with Wilcoxon Signed Ranks Test. Follow-up maintained in $6^{\text {th }}$ month, $1^{\text {st }}$ year, $2^{\text {nd }}$ year and final check-up well done clinically and radiographically to exclude any failure of implant caused by peri-implantitis. Out of the twenty failed implant in both surgical procedure which observed in the $3^{\text {rd }}$ month at stage of gingival former; no extra failure are recorded. Author thought that antibiotics can have role to prevent proceeding complications extends to failure more over patients instruction to keep good oral hygiene can play role beside.
\end{abstract}




\section{Keywords}

Antibiotics, Amoxil, Dental Implant Failure, Success, Prophylactic

\section{Introduction}

Teeth can be lost by many different causes through aging process. Preserving patient's oral health are the precedence of all dental practitioners and professionals restoring normal esthetics function speech and contour regardless atrophy, disease or injuries of stomatognathic system. Structural imbalance result from poor function can result from teeth loss making replacement after loss is integral. Dental implants are one of the most modern replacement modalities which previously include removable partial dentures, fixed partial dentures, and complete dentures. Hemchand, in his article stated that "Dental implants are made of materials which are biocompatible and they are inserted surgically into the jawbone primarily as a prosthodontics foundation" [1].

Endosteal dental implants can restore natural root position maintain boon dimensions and support comparable to that of healthy roots. This point makes implants transcend over fixed or removable prosthesis.

Long life success of dental implants is the prime goal of replacement therapy. Generally implant failure is multifactorial one of these factors is infection. Professionals in implant dentistry spend all efforts to reach the maximum standard to prevent any failures that can be caused by infection.

Late implant failure can result from either biological or mechanical causes. In this article we evaluate the candidate concerning peri-implantitis as a biological cause of failure; it usually involves the loss of hard tissue support or osseointegration [2,3]. In recent article review published by Thanh An Do, 2020 stated that resolving late failure is more difficult [4].

Dental implant procedures are varied from simple surgical process as single dental implant replacement or complicated procedures as inferior alveolar nerve transpositions and direct maxillary sinus lifting process. Theoretically; it's well-known that difficult to treat infected biomaterials and most of the infected implants have to be replaced making patient prone to another surgery after minimum three months healing process waiting [5]. For such causes, all endeavors spend to avoid microorganism contamination in the surgical site [6].

Biomaterial associated infections, occurred by oral microorganisms which play a key role in such situations as it colonized on implant surface during surgery (perioperative contamination) 
"Presence of bacterial biofilms, overheating and surgical trauma are considered the main reasons of early implant failure $[7,8] "$.

Immediate or post extraction defined as placement of dental implant at once after extraction of a tooth. Extraction should be performed in smooth delicate procedure avoiding injuries to the bone surrounding, this procedure might need antibiotics cover even in clean technique [9]. The routine use of antibiotic therapy as premedication before dental surgery is not recommended [10]. However, treatment protocol in suspected cases with infection; preferable to prescribe antibiotics as preoperative or postoperative or both to prevent any complication during healing. According to many published articles "antibiotic prophylaxis seems to be appropriate in clean contaminated surgery as implant placement procedure" [11] in a percentage ranged between $(10-15 \%)$ as expectation [12].

Conversely; there is various argue concerning aseptic surgical techniques in conjunction with antibiotics use can reduce postoperative complications as infections that can end with implant failure. Moreover, adverse effect of haphazard unauthorized antibiotics prescription as mild gastrointestinal symptoms to more serious hypersensitivity reactions as well as antibioticresistant bacteria can manifest [6].

Infected sites presence, prolonged mucosal surgeries or large foreign materials placement, in such situation antibiotics can be recommended [13]. Currently, no clear special guidelines are available for healthy patient antibiotics prescription post implant surgery. Additionally, it should be considered in previously medically compromised individuals or complicated sites which could be a main risk for infection and failure [14].

Complications such as pain, suppuration, mucositis, implant mobility, are main signs and symptoms can reflect failure; evident with radiographic bone resorption [15].

\section{Aims}

Evaluating influence of antibiotics administration on dental implants failure in medically health patients underwent simple to moderate dental implant surgeries whether delayed or immediate (post-extraction) implantation. Emphasize the pros and cons with oral antibiotics use versus no antibiotic administration in implant surgeries. According to our search no previously performed such hypothesis in the researches. 


\section{Patients and Method}

Application Form for Research is organized following the recommendation of the scientific committee of the Nineveh Health Directorate/Ministry of Health-Iraq. Human Ethical Scientific Approval Licenses number is 4101 in 2/2/2021.

One hundred twenty (120) consecutive medically fit patients necessitate oral rehabilitation by dental implants in different tooth loss area ranged from single to multiple teeth replacement (not more than 6 teeth).

Written informed consent with regard to treatment and measurement procedures was given by all patients and approval from the Research Committee in our directorate was duly obtained. All the implants came from one manufacturer (Superline, Dentium, Korea). All patients operated by same oral / maxillofacial surgeon in 2 different places (special private clinic and dental implant unit in maxillofacial department of the Al-Salam Teaching Hospital) in the period from 1/1/2018-1/12/2020. Both male and female are involved in this study.

Inclusive Criteria: Patients were included in this study are:

1. Aged from 18-50 years old

2. Medically healthy candidate

3. No history of drug allergy or drug abuse

4. Agree to share in the study

5. Agree to be followed for three years

6. Simple to moderate dental implants procedures

Patients were not admitted to the study (excluded) if any of the following criteria were present:

1. Systemically diseased patients and medically necessary antibiotic prophylaxis

2. Non-steroidal anti-inflammatory drug therapy use

3. History of antibiotic therapy 6 months prior to the study

4. Allergic patients to penicillin or other drugs

5. Pregnant ladies

6. Patients not like to share

7. Unwillingness to return for the follow-up examinations

8. Advanced implant surgeries as maxillary sinus lifting and inferior alveolar nerve translocation

9. Complicated extraction cases

10. Infected tooth before extraction

In this cohort prospective study, patients divided into two main groups:

Group one: 60 patients underwent delayed dental implant surgeries (150 implants). Previously extracted teeth 
Group two: 60 patients underwent immediate dental implant surgeries (150 implants). Immediate extraction and implantation at once are performed.

These patients were randomly allocated to 3 different categories:

1. No Antibiotic Treatment (NOAB)

2. Preoperative and Postoperative Antibiotic Treatment (PPAB) consisting of amoxicillin $1.5 \mathrm{~g} 1$ hour before surgery and $500 \mathrm{mg}$ three times per day; for 7 days following surgery

3. Postoperative Antibiotic Coverage (POAB) consisting of and $500 \mathrm{mg}$ three times per day; started after surgery and continued for 1 week after surgery. All the procedure are discussed and explained with the participant. Informed written consent for each contributor is filled and signed

\section{Patients Selection}

These patients initially presented with different edentulous sites (single or multiple teeth loss not exceed 6 teeth/4 implants) in upper and lower arches, either unilaterally or bilaterally. Currently each candidate assessed clinically, radiographically with diagnostic casts preparations before surgeries. Patients underwent full mouth preoperative scaling to keep an oral environment more favorable to wound healing. Patients instructed to rinse with chlorhexidine gluconate $0.2 \%$ solution for 1 minute at the time of surgery; before each procedure. Immediate and delayed implant placement is performed.

Clinician following the routine 2 step surgical procedure for implant placement, waiting three months for both soft and hard tissues healing process and fixed single or multiple prosthesis fabrication. Ice packs and non-steroidal anti-inflammatory drug (paracetol tablets $500 \mathrm{mg}$ twice daily for 1 day) were recommended post-surgery. Patients were also advised to keep good oral hygiene with through mouth rinsing with chlorhexidine gluconate $0.2 \%$ twice daily for 15 days following surgery and to not to brush the area of surgery for 2 weeks. Post- surgical examination are done after 3 days, 1 week, 4 weeks, 12 weeks to evaluate the soft tissues healing process for presence of any sign can reflect infections as edema, erythema, pain, heat, and exudate. As well as radiographical evaluation for bone healing assessment performed after prosthesis placement in the periods include 6 months, $1^{\text {st }}$ year, $2^{\text {nd }}$ year and $3^{\text {rd }}$ year. Implant failure was defined as loss of implant bone integration (biological failure cause).

Patients sub-grouping: patients separated into 3 groups. Each group involves 20 participants with 50 dental implants placed indifferent sites as follow:

Group A: 20 patients, 50 dental implants, no antibiotic treatment are prescribed to the patients (NOAB). 
Group B: 20 patients, 50 dental implants, preoperative and postoperative antibiotic treatment are recommended (PPAB); consisting of amoxicillin $1.5 \mathrm{~g} 1$ hour before surgery and $500 \mathrm{mg}$ three times per day/ for 7 days following surgery.

Group C: 20 patients, 50 dental implants, Postoperative Antibiotic Coverage (POAB) consisting of and $500 \mathrm{mg}$ three times per day; started after surgery and continued for 1 week after surgery.

Statistical analysis was conducted using the commercial package SPSS. Kruskal-Wallis Test for the difference of group means was applied. A p-value of 0.05 determining the effectiveness of different amoxicillin regimens was selected as the level of statistical significance with the Friedman Test and Wilcoxon Signed Ranks Test for multiple comparisons.

\section{Results}

Three hundred dental implants were seated in 120 patients; adherent to study protocol as shown in Table 1. Success and failure rate are also shown in Table 1.

Table 2 show descriptive analysis involving age, gender and complications in both delayed and direct implantation. Loss of $(10,8)$ implants are seen in delayed and immediate implantation consequently give success rate $(93.33 \%$ and $94.66 \%)$.

Kruskal-Wallis Test has been used to evaluate the correlation between complications and failure rate in both delayed and immediate implantation in two different follow-up periods ( $3^{\text {rd }}$ and $6^{\text {th }}$ month). Significant correlation is shown in both implantation groups $(0.007,0.001)$ with high significance for the delayed type in comparison to immediate post extraction implantation.

On the contrary in the $6^{\text {th }}$ month comparison no significant relation are seen as theirs is no more failure recorded in the delayed group with 2 implants recorded as failure in the immediate implantation procedure. In the follow-up periods $\left(1^{\text {st }}, 2^{\text {nd }}, 3^{\text {rd }}\right.$ years $)$ no failures recorded in both groups (Table 3).

Friedman-test used to evaluate the co-relation between antibiotic use and success rate (Table 4). No significant relation observed in both immediate PPAB use and POAB on the contrary the rest four categories show significant result.

Table 5 show Wilcoxon Signed Ranks Test description of follow-up period and antibiotics use; significant relation on antibiotics use and success rate all over the follow-up periods. 


\begin{tabular}{|c|c|c|c|c|c|c|c|}
\hline Groups & AB Use & No. Patient & No. Implant & Success / Implant & & Failure / Implant & \\
\hline \multirow[t]{3}{*}{ Delay IS } & NOAB & 20 & 50 & \multirow[t]{3}{*}{140} & \multirow[t]{3}{*}{93.33} & \multirow[t]{3}{*}{10} & \multirow[t]{3}{*}{6.66} \\
\hline & PPAB & 20 & 50 & & & & \\
\hline & POAB & 20 & 50 & & & & \\
\hline \multirow[t]{3}{*}{ Immediate IS } & NOAB & 20 & 50 & \multirow[t]{4}{*}{142} & \multirow[t]{4}{*}{94.66} & \multirow[t]{4}{*}{8} & \multirow[t]{4}{*}{5.33} \\
\hline & PPAB & 20 & 50 & & & & \\
\hline & POAB & 20 & 50 & & & & \\
\hline Total & & 120 & 300 & & & & \\
\hline
\end{tabular}

Table 1: Study protocol.

\begin{tabular}{|c|c|c|c|c|c|}
\hline \multirow{3}{*}{ Variable } & & Delayed & & Immediate & \\
\cline { 2 - 6 } Age Group & & Pt. No. & $\%$ & Pt. No. & $\%$ \\
\cline { 2 - 6 } & $20-30 \mathrm{y}$ & 24 & 40 & 34 & 56.66 \\
\cline { 2 - 6 } & $31-40 \mathrm{y}$ & 27 & 45 & 15 & 25 \\
\hline \multirow{4}{*}{ Gender } & $41-50 \mathrm{y}$ & 9 & 15 & 11 & 18.33 \\
\cline { 2 - 6 } & Male & 21 & 35 & 36 & 60 \\
\hline Complications & No Complication & 39 & 65 & 24 & 40 \\
\cline { 2 - 6 } & Pain & 4 & 65 & 41 & 68.3 \\
\cline { 2 - 6 } & Dehiscence & 1 & 1.7 & 6 & 13 \\
\cline { 2 - 6 } & Edema & 2 & 3.3 & & \\
\cline { 2 - 6 } & Swelling & 2 & 3.3 & & \\
\hline
\end{tabular}

Table 2: Descriptive analysis (age, gender and complications) in delayed and immediate implantation.

\begin{tabular}{|c|c|c|c|c|}
\hline Complication & 3 month follow-up & Ranks & Kruskal-Wallis Test & Significance \\
\hline \multirow{2}{*}{ Delayed } & Success & 140 & 0.007 & Highly significance \\
\cline { 2 - 5 } & Failure & 10 & & \\
\hline \multirow{2}{*}{ Immediate } & Success & 142 & 0.001 & \\
\cline { 2 - 5 } & Failure & 8 & & \\
\hline
\end{tabular}

Table 3: Complications and failure (Kruskal-Wallis Test) 3 month follow up.

\begin{tabular}{|c|c|c|c|c|c|c|}
\hline $\begin{array}{c}\text { Statistical } \\
\text { Analysis }\end{array}$ & $\begin{array}{c}\text { Delayed/ } \\
\text { NOAB }\end{array}$ & $\begin{array}{c}\text { Delayed// } \\
\text { PPAB }\end{array}$ & $\begin{array}{c}\text { Delayed/ } \\
\text { POAB }\end{array}$ & $\begin{array}{c}\text { Immediate/ } \\
\text { NOAB }\end{array}$ & $\begin{array}{c}\text { Immediate/ } \\
\text { PPAB }\end{array}$ & $\begin{array}{c}\text { Immediate/ } \\
\text { POAB }\end{array}$ \\
\hline $\mathbf{N}$ & 20 & 20 & 20 & 20 & 20 & 20 \\
\hline Chi-Square & 16 & 12 & 12 & 15.667 & 5.333 & 4 \\
\hline df & 4 & 4 & 4 & 4 & 4 & 4 \\
\hline Asymp. Sig. & 0.003 & 0.017 & 0.017 & 0.004 & 0.255 & 0.406 \\
\hline
\end{tabular}

Table 4: Antibiotic use and success rate (Friedman Test). 


\begin{tabular}{|c|c|c|c|c|c|}
\hline \multicolumn{7}{|c|}{ Test Statistics } \\
\hline $\mathrm{Z}$ & $3^{\text {rd }}$ Month & $6^{\text {th }}$ Month & $1^{\text {st }}$ Year & $2^{\text {nd }}$ Year & $3^{\text {rd }}$ Year \\
\hline Asymp. Sig. (2-tailed) & $-8.600-\mathrm{b}$ & $-8.725-\mathrm{b}$ & $-8.725-\mathrm{b}$ & $-8.725-\mathrm{b}$ & $-8.725-\mathrm{b}$ \\
\hline
\end{tabular}

Table 5: Correlation of antibiotics use and success in 3 years follow-up periods (Wilcoxon

Signed Ranks Test).

\section{Discussion}

This clinical study performed to show primarily the effect of antibiotics prescription in two different surgical procedures (delayed and immediate dental implantation) with various categories (no use of antibiotics, pre/postsurgery use and postsurgery use only). The advantages of antibiotics in patients undergoing implant surgery remains controversial. According to our search; limited clinical studies conflict this comparison between two surgical processes; delayed implant surgery with healed clean surgical site is achieved, on the other aspect, immediate post extraction implant surgery are performed.

Generally speaking, antibiotics start to be used in extensive manner and unwarranted with all the cons side effect as increase bacterial resistance and potential risks for the efficacy of antimicrobial therapy [16].

In a review study published by Hemchand 2016 stated that "pre- or post-operative use of antibiotics in conjunction with implant surgery and its correlation with failure and success rates are poorly documented in the literature" [1]. Excessive instruction of antibiotics used in simple and moderate cases educates essential serious assessment of exact benefit and relation with implant surgeries.

Undoubtedly bacterial contamination whilst implant insertion can end with failure. Professionals confirm that it's difficult to treat biomaterials infection based on that infected implants have to be replaced in most cases [17].

Basically as a role implant placement should be seated in strict aseptic conditions to prevent and reserve the osseointegration process.

Key surgical principle is to perform the surgical field disinfection as much as possible; this can be performed in various ways. Antiseptic mouthwashes use and wound irrigation are most commonly advised attitude use. In a study published by Hwang D, 2007; highlighting that "One of the most recommended antiseptic rinse is chlorhexidine digluconate. It has been shown that $0.12 \%$ of chlorhexidine mouthwash during immediately preoperative moment reduces the percentage of implant loss from 13.5 to $4.4 \%$ in patients with type-2 diabetes" [17].

Al-Rawee RY | Volume 2; Issue 1 (2021) | JDHOR-2(1)-023 | Clinical Study 
Chlorhexidine gluconate $0.2 \%$ solution used for 1 minute before each procedure. This maneuver can give minute sterilization of the surgical field with no effect on the exact study. According to Misch recommendation for prophylactic protocol describe 5 different categories for antibiotics used. Briefly are described in Table 6 [1].

\begin{tabular}{|c|c|c|c|c|}
\hline $\begin{array}{c}\text { Category } \\
\text { No. }\end{array}$ & Case Severity & $\begin{array}{c}\text { Patient } \\
\text { Description }\end{array}$ & Clinical Use & Instruction \\
\hline 1 & Mild & Healthy & $\begin{array}{l}\text { Infection risk is } \\
\text { low in normal } \\
\text { extractions cases }\end{array}$ & No AB Use \\
\hline 2 & Moderate & Healthy & $\begin{array}{l}\text { Extractions which } \\
\text { are traumatic or } \\
\text { socket } \\
\text { preservation } \\
\text { procedures and } \\
\text { immediate implant } \\
\text { placements }\end{array}$ & $\begin{array}{l}\text { Presurgical antibiotic loading } \\
\text { followed by a single dose of } \\
\text { antibiotic postsurgically }\end{array}$ \\
\hline 3 & $\begin{array}{l}\text { Moderate to high } \\
\text { risk }\end{array}$ & Healthy & $\begin{array}{l}\text { Multiple implants } \\
\text { with extensive } \\
\text { soft-tissue } \\
\text { reflection or } \\
\text { immediate } \\
\text { implants } \\
\text { placements along } \\
\text { with bone grafting } \\
\text { and membrane }\end{array}$ & $\begin{array}{l}\text { Preoperative loading dose of } \\
\text { antibiotics is followed by three } \\
\text { postsurgical doses for a day to be } \\
\text { continued for } 3 \text { days }\end{array}$ \\
\hline 4 & High & $\begin{array}{c}\text { Medically } \\
\text { Compromised }\end{array}$ & $\begin{array}{l}\text { Implant surgeries } \\
\text { with sinus floor } \\
\text { lifts, autogenous } \\
\text { block bone grafts }\end{array}$ & $\begin{array}{l}\text { Preoperative loading dose of } \\
\text { antibiotics is followed by three } \\
\text { postsurgical doses for a day to be } \\
\text { continued for } 5 \text { days }\end{array}$ \\
\hline 5 & High & $\begin{array}{c}\text { Medically } \\
\text { Compromised }\end{array}$ & $\begin{array}{c}\text { All sinus } \\
\text { augmentation } \\
\text { procedures }\end{array}$ & $\begin{array}{l}\text { Loading dose of antibiotics } 1 \text { day } \\
\text { prior to the surgery (ensuring } \\
\text { adequate levels in sinus tissues } \\
\text { before surgery) and a beta- } \\
\text { lactamase antibiotic to be used for } \\
5 \text { days due to the high incidence } \\
\text { of beta-lactamase pathogens in } \\
\text { maxillary sinus infections. }\end{array}$ \\
\hline
\end{tabular}

Table 6: Misch recommendation protocol for prophylactic use of antibiotics. 
In this study we exclude the category 4 and 5 were antibiotics use is mandatory and preferable to be used; so exclude interactions of variable. In delayed implants surgery; usually patient underwent simple to moderated type of surgeries even in multiple implant placement, on the contrary presence of extraction in immediate post extraction implant can complicate the implant surgeries this because extraction should be smooth delicate and skilled which in some cases can be complicated when difficult extraction manifested.

Complications as pain, edema, dehiscence and swelling occur in both procedures (15\%, $31.6 \%$ ) respectively although delayed surgeries show half percent of that immediate one but both give good success rate. Complications usually can be treated with antibiotics from data analysis there is significant correlation of antibiotics use and preventing complications which can give rise to failure (Table 4 and 5).

Preoperative prophylactic antibiotics; widely used in risk patients where bacterial endocarditis suspected, this protocol approved and recommended in 1997 by the American Heart Association (AHA). They endorsed prescription of "antimicrobial prophylaxis before any dental procedure in which bleeding can occur, including the placement of dental implants" [18]. This recommendation is agreed also by the Canadian Dental Association [19].

Amoxicillin and penicillin considered as drug of choice for treatment due to their superior absorption and prolonged serum levels [18]. Here following the roles, amoxicillin are the drug of choice to be recommended; group B instruct to use Preoperative And Postoperative Antibiotic Treatment (PPAB); consisting of amoxicillin $1.5 \mathrm{~g} 1$ hour before surgery and 500 mg three times per day/ for 7 days following surgery.

In a retrospective study published by Abdulwassie and Dhanrajani 2002 conducted for implant surgery patients [20]. "Amoxicillin $500 \mathrm{mg}$ was given thrice daily, initiating 1 day prior to surgery and continued for 10 days, and the success rate of $95.57 \%$ was achieved".

In this study high success rate also gained in both surgical procedures although we instruct patients to use for seven days.

Esposito, et al., 2010 in his published article conclude that some evidence advising preoperative oral use of $2 \mathrm{~g}$ of amoxicillin to notably diminish dental implants failure in satisfactory conditions [21]. On the contrary no evidences proof that whether postoperative antibiotics are beneficial, and which is the most effective antibiotic.

In published article prepared by Andy Kim, et al., 2020 in British Dental Journal conclude that preoperative single dose antibiotics may reduce occurrence of implant failure [22]. Observed 
overall risk reduction can support idea of prophylactic antibiotics use in implant dentistry significantly this proved in the meta-analysis study.

Dalia Khalil, 2016 publish an article highlighting Antibiotics in Implant Dentistry. She emphasized that "scientific evidence from various surgical fields including placement of dental implants shows no benefit of antibiotic prophylaxis beyond the day of surgery in uncomplicated routine cases [23-25]. Therefore, this extended antibiotic treatment is now increasingly being replaced by a single-dose antibiotic prophylaxis".

"The prophylactic use of preoperative V-penicillin was first postulated by the Branemark for dental implant surgery" [26].

In this study, high significant relation between use of antibiotics and success in delayed surgery cases are clear in comparison with immediate one. This can be contributed to the more complication recorded which respond to antibiotics use.

From what we survey in scientific published articles most of them instruct professional to recommend prophylactic antibiotics as indicated, yet clinicians still prescribing them widely. Legally this non-guided practice protocol is rejected or not accepted ethically. In some instances beginner surgeons in implant dentistry can prescript antibiotics possibly to prevent any chance of consequent failure with such high cost surgery [27].

Since 1997 Daniel publish a big analysis study of 2.641 implants with 2 stage surgery show high failure rate in patients who don't use preoperative antibiotics; with risk loss up to twice that of use antibiotics [9]. Basically on these data, there is challenge when to use antibiotics with appropriate adequate doses or not to use antibiotics. Increased costs; side effect risk as anaphylactic shock; added to that development of resistant microbial strains. For that careful evaluation of pros and cons should be kept in mind.

Reasons for implant failure have been widely discussed in the literature. In 1999 Esposito and colleagues reported that "one of the three major causes of implant failure might be infection associated with the surgical procedure itself" [28]. Accordingly antibiotics can be administered postoperatively in different surgeries.

In general it's well-known that post-operative recommendation of antibiotics specifically to prevent infection in risky patients, in extensive surgery cases, implantation of large foreign materials in the body. Recently, suggestion of short term course of antibiotics has been advised to avoid the overuse of antibiotics side effects that range from diarrhea to life-threatening allergic reactions [21]. 
In a longitudinal study prepared by Kashani 2005 stated that "A total of 868 consecutively treated patients were included in the study (403 men and 465 women) [29]. The mean age was 54.1 years (range, 15-91 years). A total of 3,021 implants were installed during the study period". The implants were installed with two step surgery, likewise patients divided into two groups: "(group I) patients received an antibiotic regimen consisting of $2 \mathrm{~g}$ phenoxymethyl penicillin twice daily for 1 week postoperatively, the first dose being given 1 hour prior to surgery on the other hand Group II; the routine changed, and patients received only one dose of phenoxymethyl penicillin ( $2 \mathrm{~g}) 1$ hour preoperatively and one dose postoperatively the same day". No osseointegration are the criteria of failure in healing process evaluation. The authors here conclude that No differences were found between the groups that received only postoperative antibiotics or received no antibiotics at all.

A recent meta-analyses article published by Cochrane Collaboration in 2019 "Cochrane Database of Systematic Reviews" stated the result of six clinical trials revealed a statistically significant higher number of participants experiencing implant failures in the group not receiving antibiotics [21].

In this study, significant correlation between success rate and postoperative use of antibiotics are clear with high significance in second stage group.

Follow-up periods post implant surgery is crucial. Researcher and implantologist in dental implant surgery considers 2-3 years follow post-surgery are enough to reflect success of the implant and long life survival.

Laskin, 2000 perform an important article conclude that "These data for 2,973 implants were recorded and correlated with failure of osseointegration during healing (Stage 1), at surgical uncovering (Stage 2), before loading the prosthesis (Stage 3), and from prosthesis loading to 36 months (Stage 4). The results showed a significantly higher survival rate at each stage of treatment in patients who had received preoperative antibiotics [30]. A significant difference in implant failure was found when comparing preoperative antibiotic coverage at any dose (4.6\% failures) and no preoperative antibiotic coverage (10\% failures)".

Significant correlation are shown in this study analysis for three years follow-up periods with wilcoxon signed ranks test. Follow-up maintained in $6^{\text {th }}$ month, $1^{\text {st }}$ year, $2^{\text {nd }}$ year and final checkup well done clinically and radiographically to exclude any failure of implant caused by peri-implantitis. Out of the twenty failed implant in both surgical procedure which observed in the $3^{\text {rd }}$ month at stage of gingival former; no extra failure are recorded. Author thought that antibiotics can have role to prevent proceeding complications extends to failure more over patients instruction to keep good oral hygiene can play role beside. 
Limitation of this study may be preferable to increase the participant number with different antibiotics regime and with different implant systems.

\section{Conclusion}

Antibiotics prescription can have significant role in implant success / survival rate at each stage of treatment in patients who had received preoperative antibiotics. A significant difference in implant failure was found when comparing antibiotic coverage in both surgery stages (delayed $93.33 \%$ and immediate placement $94.66 \%$ ) of implant. In this study, significant correlation between success rate and postoperative use of antibiotics are clear with high significance in second stage group.

\section{References}

1. Surapaneni H, Yalamanchili PS, Basha MH, Potluri S, Elisetti N, Kumar MK. Antibiotics in dental implants: A review of literature. J Pharmacy and Bioallied Sci. 2016;8(Suppl 1):S28.

2. Manor Y, Oubaid S, Mardinger O, Chaushu G, Nissan J. Characteristics of early versus late implant failure: a retrospective study. J Oral and Maxillofacial Surg. 2009;67(12):2649-52.

3. Sakka S, Baroudi K, Nassani MZ. Factors associated with early and late failure of dental implants. J Investigative and Clin Dentistry. 2012;3(4):258-61.

4. Do TA, Le HS, Shen YW, Huang HL, Fuh LJ. Risk factors related to late failure of dental implant-a systematic review of recent studies. Int Environmental Res Public Health. 2020;17(11):3931.

5. Asenjo-Lobos C, Jofre J, Cortes M, Carlos M, Asenjo-Lobos C, Jofre J, et al. Use of antibiotics in dental implant surgery: a decision based on evidence from systematic review. Int J Odontostomat. 2015;9(1):13747.

6. Esposito M, Hirsch JM, Lekholm U, Thomsen P. Biological factors contributing to failures of osseointegrated oral implants.(II). Etiopathogenesis. European journal of oral sciences. 1998;106(3):721-64.

7. Cortizo MC, Oberti TG, Cortizo MS, Cortizo AM, de Mele MA. Chlorhexidine delivery system from titanium/polybenzyl acrylate coating: evaluation of cytotoxicity and early bacterial adhesion. J Dentistry. 2012;40(4):329-37.

8. Heuer W, Stiesch M, Abraham WR. Microbial diversity of supra-and subgingival biofilms on freshly colonized titanium implant abutments in the human mouth. Euro J Clinical Microbiology and Infectious Dis. 2011;30(2):193-200.

9. Jofre J, Valenzuela D, Quintana P, Asenjo-Lobos C. Protocol for immediate implant replacement of infected teeth. Implant Dentistry. 2012;21(4):287-94.

10. Peterson LJ. Antibiotic prophylaxis against wound infections in oral and maxillofacial surgery. J Oral Maxillofacial Surg. 1990;48(6):617-20.

11. Dent CD, Olson JW, Farish SE, Bellome J, Casino AJ, Morris HF, et al. The influence of preoperative antibiotics on success of endosseous implants up to and including stage II surgery: a study of 2,641 implants. J Oral Maxillofacial Surg. 1997;55(12):19-24.

12. Resnik RR, Misch C. Prophylactic antibiotic regimens in oral implantology: rationale and protocol. Implant Dentistry. 2008;17(2):142-50.

13. Anitua E, Aguirre JJ, Gorosabel A, Barrio P, Errazquin JM, Román P, et al. A multicentre placebo-controlled randomised clinical trial of antibiotic prophylaxis for placement of single dental implants. Eur $\mathrm{J}$ Oral Implantol. 2009;2(4):283-92. 
14. Nelson S, Thomas G. Bacterial persistence in dentoalveolar bone following extraction: a microbiological study and implications for dental implant treatment. Clin Implant Dentistry and Related Res. 2010;12(4):30614.

15. Del Fabbro M, Boggian C, Taschieri S. Immediate implant placement into fresh extraction sites with chronic periapical pathologic features combined with plasma rich in growth factors: preliminary results of singlecohort study. J Oral and Maxillofacial Surg. 2009;67(11):2476-84.

16. Paluzzi RG. Antimicrobial prophylaxis for surgery. Med Clin North Am. 1993;77:427-41.

17. Hwang D, Wang HL. Medical contraindications to implant therapy: Part II: Relative contraindications. Implant Dent. 2007;16:13-23.

18. Dajani AS, Taubert KA, Wilson W, Bolger AF, Bayer A, Ferrieri P, et al. Prevention of bacterial endocarditis. Recommendations by the American Heart Association. JAMA. 1997;277:1794-801.

19. Canadian Dental Association. Which antibiotic prophylaxis guidelines for infective endocarditis should Canadian dentists follow? JCDA. 2007;73(5):401-5.

20. Abdulwassie H, Dhanrajani PJ. Diabetes mellitus and dental implants: A clinical study. Implant Dent. 2002;11:83-6.

21. Esposito M, Worthington HV, Loli V, Coulthard P, Grusovin MG. Interventions for replacing missing teeth: antibiotics at dental implant placement to prevent complications. Cochrane Database Syst Rev. 2010;(7):CD004152.

22. Kim AS, Abdelhay N, Levin L, Walters JD, Gibson MP. Antibiotic prophylaxis for implant placement: a systematic review of effects on reduction of implant failure. Br Dental J. 2020;228(12):943-51.

23. Khalil D, Lund B, Hultin M. Antibiotics in implant dentistry. Dental Implantology and Biomaterial. UK. 2016:19-38.

24. Townley WA, Baluch N, Bagher S, Maass SW, O'Neill A, Zhong T, et al. A single pre-operative antibiotic dose is as effective as continued antibiotic prophylaxis in implant-based breast reconstruction: a matched cohort study. J Plastic, Reconstructive and Aesthetic Surg. 2015;68(5):673-8.

25. Arduino PG, Tirone F, Schiorlin E, Esposito M. Single preoperative dose of prophylactic amoxicillin versus a 2-day postoperative course in dental implant surgery: A two-centre randomised controlled trial. Eur J Oral Implantol. 2015 Jun 1;8(2):143-9.

26. Adell R, Lekholm U, Rockler B, Brånemark PI. A 15-year study of Osseo integrated implants in the treatment of the edentulous jaw. Int J Oral Surg. 1981;10:387-416.

27. Ahmad N, Saad N. Effects of antibiotics on dental implants: a review. J Clin Med Res. 2012;4(1):1.

28. Esposito M, Thomsen P, Ericsson LE, Lekholm U. Histopathologic observations on early oral implant failures. Int J Oral Maxillofac Implants. 1999;14:798-810.

29. Hossein K, Dahlin C, Bengt A. Influence of different prophylactic antibiotic regimens on implant survival rate: a retrospective clinical study. Clin Implant Dentistry and Related Res. 2005;7(1):32-5.

30. Laskin DM, Dent CD, Morris HF, Ochi S, Olson JW. The influence of preoperative antibiotics on success of endosseous implants at 36 months. Annals of Periodontol. 2000;5(1):166-74. 